Article

\title{
On Certain Covering Properties and Minimal Sets of Bigeneralized Topological Spaces
}

\author{
Samer Al Ghour* and Abdullah Alhorani \\ Department of Mathematics and Statistics, Jordan University of Science and Technology, Irbid 22110, Jordan; \\ akalhorani12@sci.just.edu.jo \\ * Correspondence: algore@just.edu.jo
}

Received: 18 May 2020; Accepted: 15 June 2020; Published: 8 July 2020

\begin{abstract}
We introduce $q$-Lindelöf, $u$-Lindelöf, $p$-Lindelöf, $s$-Lindelöf, $q$-countably-compact, $u$-countably-compact, $p$-countably-compact, and s-countably-compact as new covering concepts in bigeneralized topological spaces via $q$-open sets and $u$-open sets in bigeneralized topological spaces. Relationships between them are studied. As two symmetries relationships, we show that $q$-Lindelöf and $u$-Lindelöf are equivalent concepts, and that $q$-countably-compact and $u$-countably-compact are equivalent concepts. We focus on continuity images of these covering properties. Finally, we define and investigate minimal $q$-open set, minimal $u$-open set, and minimal $s$-open sets as three new types of minimality in bigeneralized topological spaces.
\end{abstract}

Keywords: generalized topology; bigeneralized topology; Lindelof; countably compact; continuous functions; minimal sets

\section{Introduction}

We list the notations used in this paper before the conclusion section. In 1963, Kelly [1] introduced the notion of bitopological spaces as an ordered triple $(X, \tau, \gamma)$ of a set $X$ and two topologies $\tau, \gamma$, the order is important (i.e., two bitopological spaces $(X, \tau, \gamma)$ and $\left(X, \tau^{\prime}, \gamma^{\prime}\right)$ are identical if, and only if, $\tau=\tau^{\prime}$ and $\left.\gamma=\gamma^{\prime}\right)$. After Kelly's initiation of the bitopological notion, many authors generalized many topological concepts to include bitopological spaces. Let $X$ be a nonempty set and $\sigma$ be a family of subsets of $X$. Subsequently, $\sigma$ is called a generalized topology $(\mathrm{GT})$ on $X$ and $(X, \sigma)$ is a generalized topological space (GTS) [2] if $\sigma$ contains $\varnothing$ and arbitrary union of elements of $\sigma$ is an element of $\sigma$. Let $(X, \sigma)$ be a GTS and let $B \subseteq X$. Afterwards, $B$ is called a $\sigma$-open set if $B \in \sigma$. B is called a $\sigma$-closed set if $X-B$ is $\sigma$-open. $(X, \mu)$ is said to be a strong GTS [3] if $X \in \mu$. Research in GTS is still a hot area of research in which researchers introduced several types of continuity, compactness, homogeneity, and sets are extended from ordinary topological spaces to include GTSs in [4-14], and others. As a generalization of bitopological spaces, the author in [15] defined bigeneralized topological space, as follows: an ordered triple $(X, \sigma, \delta)$ of a set $X$ and two generalized topologies $\sigma$ and $\delta$ on $X$ is called a bigeneralized topological space (BGTS). A BGTS $(X, \sigma, \delta)$ is said to be strong if $(X, \sigma)$ and $(X, \delta)$ are strong. Throughout this paper, we will assume that all GTSs, as well as BGTSs, are strong.

In [15], Datta defined quasi-open sets in bitopological spaces, as follows: a subset $A$ of a bitopological space $(X, \tau, \gamma)$ is said to be quasi-open if for every $x \in A$ there exists $U \in \tau$, such that $x \in U \subseteq A$ or $V \in \gamma$, such that $x \in V \subseteq A$. The authors in [16] have introduced quasi-open sets in BGTSs, as follows: a subset $A$ of a BGTS $(X, \sigma, \delta)$ is called quasi-open ( $q$-open) if for every $x \in A$ there exists a $\mu$-open $U$, such that $x \in U \subseteq A$ or $\sigma$-open $V \in \sigma$, such that $x \in V \subseteq A$. A subset $A$ of a BGTS $(X, \sigma, \delta)$ is $u$-open if $A \in \mu \cup \sigma$. One of the main goals of the present work is to show how the 
definitions of $q$-open sets, $u$-open sets in bitopological spaces, and some of their related concepts can be modified in order to define analogous concepts in BGTSs.

In Section 2, we mainly use $q$-open sets and $u$-open sets in BGTSs to introduce and study certain covering concepts in BGTSs. Symmetric relationships between them are introduced.

In Section 3, we define two new concepts of continuity between BGTSs. We give several relationships regarding the new continuity concepts and an old continuity concept. We focus on continuity images of covering properties that are defined in Section 2.

In Section 4, we define three types of minimality in BGTSs; we give several relationships regarding them and we focus on continuity images of each of them.

\section{Covering Properties}

In this section, we mainly use $q$-open sets and $u$-open sets in BGTSs to introduce and study certain covering concepts in BGTSs. Related to the new covering concepts we will give characterizations, implications, and examples.

Definition 1 ([16]). Let $(X, \sigma, \delta)$ be a BGTS. A subset $A$ of $X$ is said to be quasi-open (q-open) if for all $x \in A$ there exists a $\mu$-open set $U$, such that $x \in U \subseteq A$, or there exists a $\sigma$-open set $V$,such that $x \in V \subseteq A$. The family of all $q$-open sets in $(X, \sigma, \delta)$ will be denoted by $q(\sigma, \delta)$. A subset $A$ of $X$ is said to be quasi-closed ( $q$-closed) if the complement of $A$ is q-open.

Theorem 1 ([16]). Let $(X, \sigma, \delta)$ be a BGTS and $A \subseteq X$. Subsequently, $A$ is $q$-open of $(X, \sigma, \delta)$ if and only if $A=U \cup V$ where $U \in \mu$ and $V \in \sigma$.

Theorem 2 ([16]). Let $(X, \sigma, \delta)$ be a BGTS. Then $q(\sigma, \delta)$ is a GT on $X$.

Finite intersection of $q$-open sets of a BGTS need not to be $q$-open in general [15]:

Example 1. In $(\mathbb{R}, \mu, \sigma)$ where $\sigma=\{\varnothing\} \cup\{(-\infty, a): a \in \mathbb{R}, a \leq 7\}$ and $\delta=\{\varnothing\} \cup\{(a, \infty): a \in \mathbb{R}, a \geq$ $0\}$ the sets $U=(-\infty, 5)$ and $V=(2, \infty)$ are q-open sets, but $U \cap V=(2,5)$ is not q-open.

Theorem 3 ([16]). Let $(X, \sigma, \delta)$ be a BGTS and $B \subseteq X$. Afterwards, $B$ is $q$-closed of $(X, \sigma, \delta)$ if and only if $B=M \cap W$ where $M$ is $\sigma$-closed and $W$ is $\delta$-closed.

Definition 2. A subset $B$ of a BGTS $(X, \sigma, \delta)$ is called $u$-open if $B \in \mu \cup \sigma$. The family of all $u$-open sets in $(X, \sigma, \delta)$ will be denoted by $u(\sigma, \delta)$.

Theorem 4. Let $(X, \sigma, \delta)$ be a BGTS and $A \subseteq X$. Then $u(\sigma, \delta) \subseteq q(\sigma, \delta)$.

Proof. Let $A \in u(\sigma, \delta)$. Then $A \in \mu$ or $A \in \sigma$. Since $\varnothing \in \mu \cap \sigma$ and $A=A \cup \varnothing$, then by Theorem 1, we have $A \in q(\sigma, \delta)$.

Theorem 5. Let $(X, \sigma, \delta)$ be a BGTS. Then $q(\sigma, \delta)$ is the smallest GT on X containing both $\mu$ and $\sigma$.

Proof. By Theorem 2, $q(\sigma, \delta)$ is a GT on X. Because $\mu \subseteq u(\sigma, \delta)$ and $\sigma \subseteq u(\sigma, \delta)$ and by Theorem 4, $u(\sigma, \delta) \subseteq q(\sigma, \delta)$, then $q(\sigma, \delta)$ containing both $\mu$ and $\sigma$. Let $\gamma$ be a GT on $X$ containing $\mu$ and $\sigma$, and let $A \in q(\sigma, \delta)$. By Theorem $1, A=U \cup V$, where $U \in \mu$ and $V \in \sigma$. Because $\mu \subseteq \gamma$ and $\sigma \subseteq \gamma$ then $U \in \gamma$ and $V \in \gamma$, and, hence, $A=U \cup V \in \gamma$. Thus $q(\sigma, \delta) \subseteq \gamma$.

Definition 3 ([17]). Let $(X, \sigma)$ be a GTS.

(a) A collection $\mathcal{F}$ of subsets of $X$ is said to be a $\sigma$-cover of $X$ if the union of the elements of $\mathcal{F}$ is equal to $X$.

(b) $A \sigma$-subcover of a $\sigma$-cover $\mathcal{F}$ is a subcollection $\mathcal{G}$ of $\mathcal{F}$ which itself is a $\sigma$-cover. 
(c) $A \sigma$-cover $\mathcal{F}$ of $X$ is said to be $\sigma$-open cover if the elements of $\mathcal{F}$ are $\sigma$-open subsets of $X$.

(d) $A \sigma$-subcover of a $\sigma$-open cover $\mathcal{F}$ is a subcollection $\mathcal{G}$ of $\mathcal{F}$ which itself is a $\sigma$-open cover.

Definition 4. Let $(X, \sigma, \delta)$ be a BGTS.

(a) A collection $\mathcal{F}$ of subsets of $X$ is called a cover of $X$ if the union of the elements of $\mathcal{F}$ is equal to $X$.

(b) A sub-cover of a cover $\mathcal{F}$ of $X$ is a subcollection $\mathcal{G}$ of $\mathcal{F}$, which itself is a cover of $X$.

(c) A cover $\mathcal{F}$ of $X$ is called q-open cover if $\mathcal{F} \subseteq q(\sigma, \delta)$.

(d) A cover $\mathcal{F}$ of $X$ is called $u$-open cover if $\mathcal{F} \subseteq u(\sigma, \delta)$.

(e) A u-open cover $\mathcal{F}$ of $X$ is called $p$-open cover if $\mathcal{F} \cap(\mu-\{\varnothing\}) \neq \varnothing$ and $\mathcal{F} \cap(\sigma-\{\varnothing\}) \neq \varnothing$.

Definition 5. A GTS $(X, \sigma)$ is said to be $\sigma$-Lindelöf if each $\sigma$-open cover of $X$ has a countable subcover.

Definition 6. Let $(X, \sigma, \delta)$ be a BGTS. Subsequently,

(a) $(X, \sigma, \delta)$ is called $q$-Lindelöf if every $q$-open cover of $X$ has a countable sub-cover.

(b) $(X, \sigma, \delta)$ is called $u$-Lindelöf if every $\mu \sigma$-open cover of $X$ has a countable sub-cover.

(c) $(X, \sigma, \delta)$ is called $p$-Lindelöf if every $p$-open cover of $X$ has a countable sub-cover.

(d) $(X, \sigma, \delta)$ is called s-Lindelöf if $(X, \mu)$ is $\mu$-Lindelöf and $(X, \sigma)$ is $\sigma$-Lindelöf.

Theorem 6. A BGTS $(X, \sigma, \delta)$ is $q$-Lindelöf if and only if it is $u$-Lindelöf.

Proof. Suppose $(X, \sigma, \delta)$ is $q$-Lindelöf and let $\mathcal{F}$ be a $u$-open cover of $X$. Subsequently, by Theorem 4 , $\mathcal{F}$ is a $q$-open cover of $X$. Since $(X, \sigma, \delta)$ is a $q$-Lindelöf, $\mathcal{F}$ has a countable subcover. Therefore, $(X, \sigma, \delta)$ is $u$-Lindelöf.

Conversely, suppose that $(X, \sigma, \delta)$ is $u$-Lindelöf and $\mathcal{F}=\left\{F_{\alpha}: \alpha \in \Delta\right\}$ be a $q$-open cover of $X$. For every $\alpha \in \Delta$, by Theorem 1, there exists $B_{\alpha} \in \sigma$ and $C_{\alpha} \in \delta$ such that $F_{\alpha}=B_{\alpha} \cup C_{\alpha}$. Set $\mathcal{G}=\left\{B_{\alpha}: \alpha \in \Delta\right\} \cup\left\{C_{\alpha}: \alpha \in \Delta\right\}$. Afterwards, $\mathcal{G}$ is a $u$-open cover of $X$. By $u$-Lindelöfeness of $(X, \sigma, \delta)$, there exist countable sets $\Delta_{1}, \Delta_{2} \subseteq \Delta$ where $\left\{B_{\alpha}: \alpha \in \Delta_{1}\right\} \cup\left\{C_{\alpha}: \alpha \in \Delta_{2}\right\}$ is a countable sub-cover of $\mathcal{G}$. It is not difficult to check that $\left\{F_{\alpha}: \alpha \in \Delta_{1} \cup \Delta_{2}\right\}$ is a countable sub-cover of $\mathcal{F}$. Thus, $(X, \sigma, \delta)$ is $q$-Lindelöf.

Theorem 7. Every u-Lindelöf BGTS is s-Lindelöf.

Proof. Let $(X, \sigma, \delta)$ be a $u$-Lindelöf BGTS.

$(X, \sigma)$ is $\sigma$-Lindelöf: let $\mathcal{F}$ be a $\sigma$-open cover of $X$. By $u$-Lindelöfeness of $(X, \sigma, \delta)$, there is countable subcover $\mathcal{F}_{1}$ of $\mathcal{F}$. Hence, $(X, \sigma)$ is $\sigma$-Lindelöf.

The proof that $(X, \delta)$ is $\delta$-Lindelöf is similar to that used in the proof of $(X, \sigma)$ is $\sigma$-Lindelöf.

Theorem 8. Every u-Lindelöf BGTS is p-Lindelöf.

Proof. Let $(X, \sigma, \delta)$ be a $u$-Lindelöf BGTS and let $\mathcal{F}$ be a $p$-open cover of $X$. Then $\mathcal{F}$ is a $u$-open cover of $X$. Since $(X, \sigma, \delta)$ is a $u$-Lindelöf, $\mathcal{F}$ has a countable subcover. Therefore, $(X, \sigma, \delta)$ is $p$-Lindelöf.

Theorem 9. Let $(X, \sigma)$ be a BGTS. Then the following are equivalent:

(a) $(X, \sigma)$ is $\sigma$-Lindelöf.

(b) $(X, \sigma, \sigma)$ is $u$-Lindelöf.

(c) $(X, \sigma, \sigma)$ is $p$-Lindelöf.

(d) $(X, \sigma, \sigma)$ is s-Lindelöf.

Proof. Straightforward. 
The following two examples will show that the concepts $s$-Lindelöf and $p$-Lindelöf are independent, and they will also show that the converse of each of Theorems 7 and 8 is not true in general:

Example 2. Consider the BGTS $(\mathbb{R}, \mu, \sigma)$ where $\mu=\{\varnothing, \mathbb{R}\} \cup\{U: U \subseteq \mathbb{Q}\}$ and $\sigma=\{\varnothing, \mathbb{R}\} \cup$ $\left\{U: U \subseteq \mathbb{Q}^{c}\right\}:$

(1) $(\mathbb{R}, \mu, \sigma)$ is not $p$-Lindelöf and by Theorem 8 , it is not $u$-Lindelöf: $\{\{x\}: x \in \mathbb{R}\}$ is a p-open cover of $\mathbb{R}$ which has no countable sub-cover.

(2) $(\mathbb{R}, \mu, \sigma)$ is s-Lindelöf: let $F$ be a $\mu$-open cover of $\mathbb{R}$. Subsequently, $\mathbb{R} \in F$ and $\{\mathbb{R}\}$ is a countable subcover of $F$. Therefore, $(\mathbb{R}, \mu)$ is $\mu$-Lindelof. Similarly, we can see that $(\mathbb{R}, \sigma)$ is $\sigma$-Lindelof.

Example 3. Consider the BGTS $(\mathbb{R}, \mu, \sigma)$, where $\mu=\{\varnothing, \mathbb{R}\} \cup\left\{\mathbb{Q} \cup U: U \subseteq \mathbb{Q}^{c}\right\}$, and $\sigma=\left\{\varnothing, \mathbb{Q}^{c}, \mathbb{R}\right\}$. Then $(\mathbb{R}, \mu, \sigma)$ is $p$-Lindelöf but not s-Lindelöf and by Theorem 7 , it is not u-Lindelöf.

Proof. $(\mathbb{R}, \mu, \sigma)$ is $p$-Lindelöf: $(\mathbb{R}, \mu, \sigma)$ is $p$-compact: let $\mathcal{F}$ be a $p$-open cover of $\mathbb{R}$. Because $F \cap$ $(\sigma-\{\varnothing\}) \neq \varnothing, \mathbb{R} \in \mathcal{F}$ or $\mathbb{Q}^{c} \in \mathcal{F}$.

Case 1. $\mathbb{R} \in \mathcal{F}$. Subsequently, $\{\mathbb{R}\}$ is a countable sub-cover of $\mathcal{F}$ and we are done.

Case 2. $\mathbb{Q}^{c} \in \mathcal{F}$ and $\mathbb{R} \notin F$. Choose $U \in \mathcal{F}$, such that $0 \in U$. Afterwards, $\mathbb{Q} \subseteq U$ and so $\left\{U, \mathbb{Q}^{c}\right\}$ is a countable sub-cover of $\mathcal{F}$.

$(\mathbb{R}, \mu, \sigma)$ is not $s$-Lindelof: $\left\{\mathbb{Q} \cup\{x\}: x \in \mathbb{Q}^{c}\right\}$ is a $\mu$-open cover of $X$, which has no countable subcover. This implies that $(\mathbb{R}, \mu)$ is not $\mu$-Lindelöf and, hence, $(\mathbb{R}, \mu, \sigma)$ is not $s$-Lindelöf.

The converse of Theorem 7 is true for an s-Lindelöf BGTS, as in the following result:

Theorem 10. Let $(X, \sigma, \delta)$ be a BGTS. Subsequently, the following are equivalent:

(a) $(X, \sigma, \delta)$ is u-Lindelöf.

(b) $(X, \sigma, \delta)$ is s-Lindelöf and $p$-Lindelöf.

Proof. (a) $\Longrightarrow$ (b) Theorems 7 and 8 .

(b) $\Longrightarrow$ (a) Let $\mathcal{F}$ be $u$-open cover of $X$. We have two cases:

Case 1. $\mathcal{F}$ is a $p$-open cover of $X$. Subsequently, $\mathcal{F}$ has a countable subcover of $X$ because $(X, \sigma, \delta)$ is $p$-Lindelöf.

Case 2. $\mathcal{F}$ is not a $p$-open cover of $X$. Afterwards, $\mathcal{F}$ is either a $\sigma$-open cover of $X$ or a $\sigma$-open cover of $X$. Because $(X, \sigma)$ is $\sigma$-Lindelöf and $(X, \sigma)$ is $\sigma$-Lindelöf, in either of the two cases $\mathcal{F}$ will have a countable subcover.

Definition 7. A GTS $(X, \sigma)$ is said to be $\sigma$-countably-compact if each countable $\sigma$-open cover of $X$ has a finite subcover.

Definition 8. Let $(X, \sigma, \delta)$ be a BGTS. Then

(a) $(X, \sigma, \delta)$ is called q-countably-compact if every countable $q$-open cover of $X$ has a finite subcover.

(b) $(X, \sigma, \delta)$ is called $u$-countably-compact if every countable u-open cover of $X$ has a finite subcover.

(c) $(X, \sigma, \delta)$ is called p-countably-compact if every countable p-open cover of $X$ has a finite subcover.

(d) $(X, \sigma, \delta)$ is called s-countably-compact if $(X, \sigma)$ is $\sigma$-countably-compact and $(X, \delta)$ is $\delta$-countably-compact.

Theorem 11. A BGTS $(X, \sigma, \delta)$ is q-countably-compact if and only if it is u-countably-compact. 
Proof. Suppose that $(X, \sigma, \delta)$ is $q$-countably-compact and let $\mathcal{F}$ be a countable $u$-open cover of $X$. Afterwards, by Theorem $4, \mathcal{F}$ is a countable $q$-open cover of $X$. Since $(X, \sigma, \delta)$ be a $q$-countably-compact, $\mathcal{F}$ has a finite subcover. Therefore, $(X, \sigma, \delta)$ is $u$-countably-compact.

Conversely, suppose that $(X, \sigma, \delta)$ is $u$-countably-compact. Let $\mathcal{F}=\left\{F_{\alpha}: \alpha \in \Delta\right\}$ be a countable $q$-open cover of $X$. By Theorem 1, for each $\alpha \in \Delta$, there is $A_{\alpha} \in \mu$ and $B_{\alpha} \in \sigma$, such that $F_{\alpha}=A_{\alpha} \cup B_{\alpha}$. Set $\mathcal{G}=\left\{A_{\alpha}: \alpha \in \Delta\right\} \cup\left\{B_{\alpha}: \alpha \in \Delta\right\}$. Afterwards, $\mathcal{G}$ is a countable $u$-open cover of $X$. Since $(X, \sigma, \delta)$ is $u$-countably-compact, there exist countable sets $\Delta_{1}, \Delta_{2} \subseteq \Delta$, such that $\left\{A_{\alpha}: \alpha \in \Delta_{1}\right\} \cup\left\{B_{\alpha}: \alpha \in \Delta_{2}\right\}$ is a finite sub-cover of $\mathcal{G}$. It is not difficult to check that $\left\{F_{\alpha}: \alpha \in \Delta_{1} \cup \Delta_{2}\right\}$ is a finite subcover of $\mathcal{F}$. Thus, $(X, \sigma, \delta)$ is $q$-countably-compact.

Theorem 12. Every u-countably-compact BGTS is s-countably-compact.

Proof. Let $(X, \sigma, \delta)$ be a $u$-countably-compact BGTS. To see that $(X, \sigma)$ is $\sigma$-countably-compact, let $\mathcal{F}$ be a countable $\sigma$-open cover of X. Subsequently, $\mathcal{F}$ is a countable $u$-open cover of $X$. Because $(X, \sigma, \delta)$ is $u$-countably-compact, $\mathcal{F}$ has a finite sub-cover $\mathcal{F}_{1}$. Therefore, $\mathcal{F}_{1}$ is a finite $\mu$-sub-cover of $\mathcal{F}$. Hence, $(X, \sigma)$ is $\sigma$-countably-compact.

The proof that $(X, \delta)$ is $\delta$-countably-compact is similar to that used in the proof of $(X, \sigma)$ is $\sigma$-countably-compact.

Theorem 13. Every u-countably-compact BGTS is p-countably-compact.

Proof. Let $(X, \sigma, \delta)$ be a $u$-countably-compact BGTS and let $\mathcal{F}$ be a countable $p$-open cover of $X$. Subsequently, $\mathcal{F}$ is a countable $u$-open cover of $X$. Because $(X, \sigma, \delta)$ is $u$-countably-compact, $\mathcal{F}$ has a finite sub-cover. Therefore, $(X, \sigma, \delta)$ is $p$-countably-compact.

Theorem 14. Let $(X, \sigma)$ be a BGTS. Subsequently, the following are equivalent:

(a) $(X, \sigma)$ is $\sigma$-countably-compact.

(b) $(X, \sigma, \sigma)$ is u-countably-compact.

(c) $(X, \sigma, \sigma)$ is p-countably-compact.

(d) If $(X, \sigma, \sigma)$ is s-countably-compact.

Proof. Straightforward.

The following two examples will show that the concepts s-countably-compact and $p$-countably-compact are independent, and they will also show that the converse of each of Theorems 12 and 13 is not true in general:

Example 4. Let $X=E \cup O$ where $E$ and $O$ are the set of even natural numbers and the set of odd natural numbers, respectively. Consider the BGTS $(X, \sigma, \delta)$, where $\sigma=\{\varnothing, X\} \cup\{U: U \subseteq E\}$ and $\delta=\{\varnothing, X\} \cup$ $\{U: U \subseteq O\}$. Subsequently,

(1) $(X, \sigma, \delta)$ is not $p$-countably-compact and, by Theorem 13, it is not u-countably compact: $\{\{x\}: x \in X\}$ is a countable p-open cover of $X$, which has no finite sub-cover.

(2) $(X, \sigma, \delta)$ is s-countably compact: let $\mathcal{F}$ be a countable $\sigma$-open cover $X$. Afterwards, $X \in \mathcal{F}$ and $\{X\}$ is a finite sub-cover of $\mathcal{F}$. Therefore, $(X, \sigma)$ is $\sigma$-countably-compact. Similarly, we can see that $(X, \delta)$ is $\delta$-countably-compact.

Example 5. Consider the BGTS $(X, \sigma, \delta)$, where $X$, as in Example 4, $\sigma=\{\varnothing, X\} \cup\{E \cup U: U \subseteq O\}$, and $\delta=\{\varnothing, O, X\}$. Afterwards, $(X, \sigma, \delta)$ is p-countably-compact, but not s-countably-compact and, by Theorem 13, it is not u-countably-compact. 
Proof. $(X, \sigma, \delta)$ is $p$-countably-compact: let $\mathcal{F}$ be a countable $p$-open cover of $X$. Since $\mathcal{F} \cap(\delta-\{\varnothing\}) \neq$ $\varnothing, X \in \mathcal{F}$ or $O \in \mathcal{F}$.

Case 1. $X \in \mathcal{F}$. Subsequently, $\{X\}$ is a finite sub-cover of $\mathcal{F}$ and we are done.

Case 2. $O \in \mathcal{F}$ and $X \notin \mathcal{F}$. Choose $U \in \mathcal{F}$, such that $2 \in U$. Afterwards, $E \subseteq U$ and so $\{O, U\}$ is a finite subcover of $\mathcal{F}$.

$(X, \sigma, \delta)$ is not s-countably-compact: $\{E \cup\{x\}: x \in O\}$ is a countable $\sigma$-open cover of $X$, which has no finite sub-cover. This implies that $(X, \sigma)$ is not $\sigma$-countably-compact and, hence, $(X, \sigma, \delta)$ is not s-countably-compact.

The converse of Theorem 13 is true for s-countably-compact BGTS, as in the following result:

Theorem 15. Let $(X, \sigma, \delta)$ be a BGTS. Afterwards, the following are equivalent:

(a) $(X, \sigma, \delta)$ is u-countably-compact.

(b) $(X, \sigma, \delta)$ is s-countably-compact and p-countably-compact.

Proof. (a) $\Longrightarrow$ (b) Theorems 12 and 13 .

(b) $\Longrightarrow$ (a) Let $\mathcal{F}$ be a countable $u$-open cover of $X$. We have two cases:

Case 1. $\mathcal{F}$ is a countable $p$-open cover of $X$. Afterwards, it has a finite subcover of $X$, because $(X, \sigma, \delta)$ is $p$-countably-compact.

Case 2. $\mathcal{F}$ is not a $p$-open cover of $X$. Subsequently, $\mathcal{F}$ is either a countable $\sigma$-open cover of $X$ or a countable $\delta$-open cover of $X$. Because $(X, \sigma)$ is $\sigma$-countably-compact and $(X, \sigma)$ is $\sigma$-countably-compact, in either of the two cases $\mathcal{F}$ will have a finite subcover.

\section{Continuity}

In this section, we define two new concepts of continuity between BGTSs. We will give several relationships regarding the two new continuity concepts and an old continuity concept. We focus on continuity images of covering properties that are defined in Section 2.

Definition 9 ([18]). A function $g:(Y, \sigma) \longrightarrow(Z, \delta)$ is called $(\sigma, \delta)$-continuous if for all $H \in \delta, g^{-1}(H) \in \sigma$.

Definition 10. A function $g:\left(Y, \sigma_{1}, \delta_{1}\right) \longrightarrow\left(Z, \sigma_{2}, \delta_{2}\right)$ is called

(a) q-continuous [16] if for all $H \in q\left(\sigma_{2}, \delta_{2}\right), g^{-1}(H) \in q\left(\sigma_{1}, \delta_{1}\right)$.

(b) $u$-continuous if for all $H \in u\left(\sigma_{2}, \delta_{2}\right), g^{-1}(H) \in u\left(\sigma_{1}, \delta_{1}\right)$.

(c) s-continuous if $g:\left(Y, \sigma_{1}\right) \longrightarrow\left(Z, \sigma_{2}\right)$ is $\left(\sigma_{1}, \sigma_{2}\right)$-continuous and $g:\left(Y, \delta_{1}\right) \longrightarrow\left(Z, \delta_{2}\right)$ is $\left(\delta_{1}, \delta_{2}\right)$-continuous.

Theorem 16. For any function $g:\left(Y, \sigma_{1}, \delta_{1}\right) \longrightarrow\left(Z, \sigma_{2}, \delta_{2}\right)$ between the BGTSs $\left(Y, \sigma_{1}, \delta_{1}\right)$ and $\left(Z, \sigma_{2}, \delta_{2}\right)$, the following are equivalent:

(a) $g$ is q-continuous.

(b) $g:\left(Y, q\left(\sigma_{1}, \delta_{1}\right)\right) \longrightarrow\left(Z, q\left(\sigma_{2}, \delta_{2}\right)\right)$ is $\left(q\left(\sigma_{1}, \delta_{1}\right), q\left(\sigma_{2}, \delta_{2}\right)\right)$-continuous.

(c) For all $H \in u\left(\sigma_{2}, \delta_{2}\right), g^{-1}(H) \in q\left(\sigma_{1}, \delta_{1}\right)$.

Proof. (a) $\Longleftrightarrow$ (b) Obvious.

(a) $\Longrightarrow$ (c) Follows from the definition and Theorem 4 .

(c) $\Longleftarrow$ (a) Suppose $g^{-1}(H) \in q\left(\sigma_{1}, \delta_{1}\right)$ for all $H \in u\left(\sigma_{2}, \delta_{2}\right)$ and let $G \in q\left(\sigma_{2}, \delta_{2}\right)$. By Theorem $1, G=A \cup B$ where $A \in \sigma_{2}$ and $B \in \delta_{2}$. By assumption $g^{-1}(A) \in q\left(\sigma_{1}, \delta_{1}\right)$ and $g^{-1}(B) \in q\left(\sigma_{1}, \delta_{1}\right)$. Thus, by Theorem $2, g^{-1}(A \cup B)=g^{-1}(A) \cup g^{-1}(B) \in q\left(\sigma_{1}, \delta_{1}\right)$. Therefore, $g$ is $q$-continuous.

Corollary 1. u-continuous functions between BGTSs are q-continuous. 
Proof. Follows from Theorems 2 and 16.

The converse of Corollary 1 is not true in general, as can be seen from the following example:

Example 6. Let $Y=Z=\mathbb{R}, \sigma_{1}=\{\varnothing, \mathbb{R}\} \cup\{(-\infty, a): a \in \mathbb{R}\}$ and $\delta_{1}=\sigma_{2}=\delta_{2}=\{\varnothing, \mathbb{R}\} \cup$ $\{(a, \infty): a \in \mathbb{R}\}$. Define $g:\left(Y, \sigma_{1}, \delta_{1}\right) \longrightarrow\left(Z, \sigma_{2}, \delta_{2}\right)$ by $g(y)=y^{2}$. Then

(1) $g$ is q-continuous: let $H \in q\left(\sigma_{2}, \delta_{2}\right)=\delta_{2}$. Then

$$
g^{-1}(H)=\left\{\begin{array}{cc}
\mathbb{R} & \text { if } H=\mathbb{R} \text { or } H=(b, \infty) \text { where } b<0 \\
(-\infty,-\sqrt{b}) \cup(\sqrt{b}, \infty) & \text { if } H=(b, \infty) \text { where } b \geq 0 \\
\varnothing & \text { if } H=\varnothing
\end{array} .\right.
$$

Therefore, $g^{-1}(H) \in q\left(\sigma_{1}, \delta_{1}\right)$.

(2) $g$ is not $u$-continuous: Since $(0, \infty) \in u\left(\sigma_{2}, \delta_{2}\right)$ while $g^{-1}((0, \infty))=(-\infty, 0) \cup(0, \infty) \notin u\left(\sigma_{1}, \delta_{1}\right)$.

Theorem 17. A function $g:\left(Y, \sigma_{1}, \delta_{1}\right) \longrightarrow\left(Z, \sigma_{2}, \delta_{2}\right)$ is s-continuous if and only if $g^{-1}(H) \in \sigma_{1}$ for all $H \in \sigma_{2}$ and $g^{-1}(H) \in \delta_{1}$ for all $H \in \delta_{2}$.

Proof. Straightforward.

Corollary 2. s-continuous functions between BGTSs are u-continuous.

The converse of Corollary 2 is not true in general, as it can be seen from the following example:

Example 7. Let $Y=Z=\mathbb{R}, \sigma_{1}=\delta_{2}=\{\varnothing, \mathbb{R}\} \cup\{(-\infty, a): a \in \mathbb{R}\}$ and $\delta_{1}=\sigma_{2}=\{\varnothing, \mathbb{R}\} \cup$ $\{(a, \infty): a \in \mathbb{R}\}$. Define $g:\left(Y, \sigma_{1}, \delta_{1}\right) \longrightarrow\left(Z, \sigma_{2}, \delta_{2}\right)$ by $g(y)=y$. Then

(1) $g$ is u-continuous: let $H \in u\left(\sigma_{2}, \delta_{2}\right)=\sigma_{2} \cup \delta_{2}=\delta_{1} \cup \sigma_{1}=\sigma_{1} \cup \delta_{1}=u\left(\sigma_{1}, \delta_{1}\right)$. Subsequently, $g^{-1}(H)=H \in u\left(\sigma_{1}, \delta_{1}\right)$.

(2) $g$ is not s-continuous: since $(1, \infty) \in \sigma_{2}$ while $g^{-1}((1, \infty))=(1, \infty) \notin \sigma_{1}$, then $g:\left(Y, \sigma_{1}\right) \longrightarrow\left(Z, \sigma_{2}\right)$ is not $\left(\sigma_{1}, \sigma_{2}\right)$-continuous. Therefore, $g$ is not s-continuous.

Theorem 18. The q-continuous image of a q-Lindelöf BGTS is q-Lindelöf.

Proof. Let $g:\left(Y, \sigma_{1}, \delta_{1}\right) \longrightarrow\left(Z, \sigma_{2}, \delta_{2}\right)$ be $q$-continuous and surjective with $\left(Y, \sigma_{1}, \delta_{1}\right)$ is $q$-Lindelöf. Let $\mathcal{F}$ be a $q$-open cover of $Z$. By $q$-continuity of $g, g^{-1}(F) \in q\left(\sigma_{1}, \delta_{1}\right)$ for all $F \in \mathcal{F}$. Put $\mathcal{H}=\left\{g^{-1}(F): F \in \mathcal{F}\right\}$. Because

$$
\begin{aligned}
Y & =g^{-1}(Z) \\
& =g^{-1}(\bigcup\{F: F \in \mathcal{F}\}) \\
& =\bigcup\left\{g^{-1}(F): F \in \mathcal{F}\right\}
\end{aligned}
$$

then $\mathcal{H}$ is a $q$-open cover of $Y$. Since $\left(Y, \sigma_{1}, \delta_{1}\right)$ is $q$-Lindelöf $\mathcal{H}$ has a countable subcover $\mathcal{H}_{1}$. Choose a countable collection $\mathcal{F}_{1} \subseteq \mathcal{F}$ with $\mathcal{H}_{1}=\left\{g^{-1}(F): F \in \mathcal{F}_{1}\right\}$. By surjectivity of $g$

$$
\begin{aligned}
Z & =g(Y) \\
& =g\left(\bigcup\left\{g^{-1}(F): F \in \mathcal{F}_{1}\right\}\right) \\
& =\bigcup\left\{g\left(g^{-1}(F)\right): F \in \mathcal{F}_{1}\right\} \\
& =\bigcup\left\{F: F \in \mathcal{F}_{1}\right\} .
\end{aligned}
$$


Therefore, $\mathcal{F}_{1}$ is a countable sub-cover of $\mathcal{F}$ and, hence, $\left(Z, \sigma_{2}, \delta_{2}\right)$ is $q$-Lindelöf.

Corollary 3. The u-continuous image of a $u$-Lindelöf BGTS is u-Lindelöf.

Proof. Theorems 6 and 18, and Corollary 1.

Theorem 19. The s-continuous image of a $p$-Lindelöf BGTS is $p$-Lindelöf.

Proof. Let $g:\left(Y, \sigma_{1}, \delta_{1}\right) \longrightarrow\left(Z, \sigma_{2}, \delta_{2}\right)$ be $s$-continuous and surjective with $\left(Y, \sigma_{1}, \delta_{1}\right)$ is $p$-Lindelöf. Let $\mathcal{F}$ be a $p$-open cover of $Z$. By Corollary $2, g$ is $u$-continuous. Therefore, $g^{-1}(F) \in u\left(\sigma_{1}, \delta_{1}\right)$ for all $F \in \mathcal{F}$. Put $\mathcal{H}=\left\{g^{-1}(F): F \in \mathcal{F}\right\}$. Since

$$
\begin{aligned}
Y & =g^{-1}(Z) \\
& =g^{-1}(\bigcup\{F: F \in \mathcal{F}\}) \\
& =\bigcup\left\{g^{-1}(F): F \in \mathcal{F}\right\},
\end{aligned}
$$

then $\mathcal{H}$ is a $u$-open cover of $Y$. Since $\mathcal{F}$ is $p$-open cover, there exist $A \in \mathcal{F} \cap\left(\sigma_{2}-\{\varnothing\}\right)$ and $B \in$ $\mathcal{F} \cap\left(\delta_{2}-\{\varnothing\}\right)$. By s-continuity of $g, g^{-1}(A) \in \sigma_{1}$ and $g^{-1}(B) \in \delta_{1}$. By surjectivity of $g, g^{-1}(A) \in$ $\mathcal{H} \cap\left(\sigma_{1}-\{\varnothing\}\right)$ and $g^{-1}(B) \in \mathcal{H} \cap\left(\sigma_{1}-\{\varnothing\}\right)$. Therefore, $\mathcal{H}$ is a $p$-open cover of $Y$. Since $\left(Y, \sigma_{1}, \delta_{1}\right)$ is $p$-Lindelöf $\mathcal{H}$ has a countable subcover $\mathcal{H}_{1}$. There exists a countable subfamily $\mathcal{F}_{1} \subseteq \mathcal{F}$ such that $\mathcal{H}_{1}=\left\{g^{-1}(F): F \in \mathcal{F}_{1}\right\}$. By surjectivity of $g$,

$$
\begin{aligned}
Z & =g(Y) \\
& =g\left(\bigcup\left\{g^{-1}(F): F \in \mathcal{F}_{1}\right\}\right) \\
& =\bigcup\left\{g\left(g^{-1}(F)\right): F \in \mathcal{F}_{1}\right\} \\
& =\bigcup\left\{F: F \in \mathcal{F}_{1}\right\} .
\end{aligned}
$$

Therefore, $\mathcal{F}_{1}$ is a countable sub-cover of $\mathcal{F}$ and, hence, $\left(Z, \sigma_{2}, \delta_{2}\right)$ is $p$-Lindelöf.

Theorem 20. The q-continuous image of a q-countably-compact BGTS is q-countably-compact.

Proof. Let $g:\left(Y, \sigma_{1}, \delta_{1}\right) \longrightarrow\left(Z, \sigma_{2}, \delta_{2}\right)$ be $q$-continuous and surjective with $\left(Y, \sigma_{1}, \delta_{1}\right)$ is $q$-countably-compact. Let $\mathcal{F}$ be a countable $q$-open cover of $Z$. By $q$-continuity of $g, g^{-1}(F) \in q\left(\sigma_{1}, \delta_{1}\right)$ for all $F \in \mathcal{F}$. Put $\mathcal{H}=\left\{g^{-1}(F): F \in \mathcal{F}\right\}$. Because

$$
\begin{aligned}
Y & =g^{-1}(Z) \\
& =g^{-1}(\bigcup\{F: F \in \mathcal{F}\}) \\
& =\bigcup\left\{g^{-1}(F): F \in \mathcal{F}\right\},
\end{aligned}
$$

then $\mathcal{H}$ is a countable $q$-open cover of $Y$. Since $\left(Y, \sigma_{1}, \delta_{1}\right)$ is $q$-countably-compact $\mathcal{H}$ has a finite subcover $\mathcal{H}_{1}$. Choose a finite collection $\mathcal{F}_{1} \subseteq \mathcal{F}$ with $\mathcal{H}_{1}=\left\{g^{-1}(F): F \in \mathcal{F}_{1}\right\}$. By surjectivity of $g$

$$
\begin{aligned}
Z & =g(Y) \\
& =g\left(\bigcup\left\{g^{-1}(F): F \in \mathcal{F}_{1}\right\}\right) \\
& =\bigcup\left\{g\left(g^{-1}(F)\right): F \in \mathcal{F}_{1}\right\} \\
& =\bigcup\left\{F: F \in \mathcal{F}_{1}\right\} .
\end{aligned}
$$

Therefore, $\mathcal{F}_{1}$ is a finite sub-cover of $\mathcal{F}$ and, hence, $\left(Z, \sigma_{2}, \delta_{2}\right)$ is $q$-countably-compact. 
Corollary 4. The u-continuous image of a u-countably-compact BGTS is u-countably-compact.

Proof. Theorems 11 and 20, and Corollary 1.

Theorem 21. The s-continuous image of a p-countably-compact BGTS is p-countably-compact.

Proof. Let $g:\left(Y, \sigma_{1}, \delta_{1}\right) \longrightarrow\left(Z, \sigma_{2}, \delta_{2}\right)$ be $s$-continuous and surjective with $\left(Y, \sigma_{1}, \delta_{1}\right)$ is $p$-countably-compact. Let $\mathcal{F}$ be a countable $p$-open cover of $Z$. By Corollary $2, g$ is $u$-continuous. Therefore, $g^{-1}(F) \in u\left(\sigma_{1}, \delta_{1}\right)$ for all $F \in \mathcal{F}$. Put $\mathcal{H}=\left\{g^{-1}(F): F \in \mathcal{F}\right\}$. Because

$$
\begin{aligned}
Y & =g^{-1}(Z) \\
& =g^{-1}(\bigcup\{F: F \in \mathcal{F}\}) \\
& =\bigcup\left\{g^{-1}(F): F \in \mathcal{F}\right\},
\end{aligned}
$$

then $\mathcal{H}$ is a countable $u$-open cover of $Y$. Since $\mathcal{F}$ is $p$-open cover, there exist $A \in \mathcal{F} \cap\left(\sigma_{2}-\{\varnothing\}\right)$ and $B \in \mathcal{F} \cap\left(\delta_{2}-\{\varnothing\}\right)$. By s-continuity of $g, g^{-1}(A) \in \sigma_{1}$ and $g^{-1}(B) \in \delta_{1}$. By the surjectivity of $g, g^{-1}(A) \in \mathcal{H} \cap\left(\sigma_{1}-\{\varnothing\}\right)$ and $g^{-1}(B) \in \mathcal{H} \cap\left(\sigma_{1}-\{\varnothing\}\right)$. Therefore, $\mathcal{H}$ is a countable $p$-open cover of $Y$. Because $\left(Y, \sigma_{1}, \delta_{1}\right)$ is $p$-countably-compact $\mathcal{H}$ has a finite subcover $\mathcal{H}_{1}$. There exists a finite subfamily $\mathcal{F}_{1} \subseteq \mathcal{F}$ such that $\mathcal{H}_{1}=\left\{g^{-1}(F): F \in \mathcal{F}_{1}\right\}$. By surjectivity of $g$,

$$
\begin{aligned}
Z & =g(Y) \\
& =g\left(\bigcup\left\{g^{-1}(F): F \in \mathcal{F}_{1}\right\}\right) \\
& =\bigcup\left\{g\left(g^{-1}(F)\right): F \in \mathcal{F}_{1}\right\} \\
& =\bigcup\left\{F: F \in \mathcal{F}_{1}\right\} .
\end{aligned}
$$

Therefore, $\mathcal{F}_{1}$ is a finite subcover of $\mathcal{F}$ and, hence, $\left(Z, \sigma_{2}, \delta_{2}\right)$ is $p$-countably-compact.

\section{Minimality}

In this section, we define types of minimality in BGTSs, we give several relationships regarding them, and we focus on continuity images of each of them.

Definition 11 ([18]). A nonempty $\sigma$-open subset $B$ of a $G T S(Y, \sigma)$ is said to be a minimal $\mu$-open set if the only nonempty $\sigma$-open set, which is contained in $B$ is $B$.

Definition 12. Let $(Y, \sigma, \delta)$ be a BGTS and B a nonempty subset of $Y$. Subsequently, B is called a

(a) minimal q-open set if $B$ is q-open and the only nonempty q-open set which is contained in $B$ is $B$.

(b) minimal u-open set if $B$ is u-open and the only nonempty u-open set that is contained in $B$ is $B$.

(c) minimal s-open set if $B$ is a minimal $\sigma$-open in $(Y, \sigma)$ and $B$ is a minimal $\delta$-open in $(Y, \delta)$.

Theorem 22. A subset $B$ of a BGTS $(Y, \sigma, \delta)$ is a minimal $q$-open set if and only if it is a minimal $q(\sigma, \delta)$-open set in $(Y, q(\sigma, \delta))$.

Proof. Straightforward.

Lemma 1. If $B$ is a minimal q-open set in a bi-generalized topological space $(Y, \sigma, \delta)$, then $B \in u(\sigma, \delta)$.

Proof. By Theorem $1, B=U \cup V$ where $U \in \sigma$ and $V \in \delta$. By Theorem $4, U \in q(\sigma, \delta)$. Since $U \in$ $q(\sigma, \delta), U \subseteq B$ and $B$ is a minimal $q$-open set in $(Y, \sigma)$, then $U=\varnothing$ or $U=B$. Similarly, we can 
conclude that $V=\varnothing$ or $V=B$. Since $B \neq \varnothing, U \neq \varnothing$ or $V \neq \varnothing$. Therefore, $B=U$ or $B=V$. In both cases, $B \in \sigma \cup \delta=u(\sigma, \delta)$.

Theorem 23. Let $(Y, \sigma, \delta)$ be a BGTS and $B \subseteq Y$. Subsequently, $B$ is a minimal $q$-open set if and only if $B$ is a minimal $u$-open set.

Proof. Necessity. Suppose that $B$ is a minimal $q$-open set. By Lemma $1, B$ is a nonempty $u$-open set. Let $C \in u(\sigma, \delta)-\{\varnothing\}$ with $C \subseteq B$. By Theorem $4, C \in q(\sigma, \delta)$. Since $B$ is minimal $q$-open, then $C=B$. It follows that $B$ is a minimal $u$-open set.

Sufficiency. Suppose that $B$ is a minimal $u$-open set. Subsequently, by Theorem $4, B$ is a non-empty $u$-open set. Let $C \in q(\sigma, \delta)-\{\varnothing\}$ with $C \subseteq B$. Because $C$ is $q$-open then, by Theorem $1, C=U \cup V$ where $U \in \sigma \subseteq u(\sigma, \delta)$ and $V \in \delta \subseteq u(\sigma, \delta)$. Since $U \in u(\sigma, \delta), U \subseteq C \subseteq B$, and $B$ is a minimal $u$-open set, then $U=\varnothing$ or $U=B$. Similarly, we can conclude that $V=\varnothing$ or $V=B$. Because $C \neq \varnothing, U \neq \varnothing$ or $V \neq \varnothing$. Thus, $U=B$ or $V=B$ and, hence, $C=B$. It follows that $B$ is a minimal $q$-open set.

Theorem 24. Let $(Y, \sigma, \delta)$ be a BGTS and $B \subseteq Y$. If $B$ is a minimal s-open set, then $B$ is a minimal u-open set.

Proof. Suppose that $B$ is a minimal s-open set. Subsequently, $B$ is nonempty and $B \in \sigma \cap \delta \subseteq u(\sigma, \delta)$. Let $C \in u(\sigma, \delta)-\{\varnothing\}$ with $C \subseteq B$. Afterwards, $C \in \sigma$ or $C \in \delta$. If $C \in \sigma$, then, as $B$ is a minimal $\sigma$-open set, $C=B$. If $C \in \delta$, then as $B$ is a minimal $\delta$-open set, $C=B$. Therefore, $B$ is a minimal $u$-open set.

Remark 1. The converse of Theorem 24 is not true in general; indeed, in Example $3 \mathbb{Q}$ is a minimal u-open set that is not a minimal s-open set.

Theorem 25. Let $(Y, \sigma, \delta)$ be a BGTS and B a minimal u-open set in $(Y, \sigma, \delta)$. Then

(a) If $B \in \sigma$, then $B$ is a minimal $\sigma$-open set.

(b) If $B \in \delta$ then $B$ is a minimal $\delta$-open set.

(c) If $B \in \sigma \cap \delta$, then $B$ is a minimal s-open set.

Proof. (a) Suppose that $B$ is a minimal $u$-open set and $B \in \sigma$. Let $B \in \sigma-\{\varnothing\}$ with $C \subseteq B$. Since $B$ is minimal $u$-open and $C \in \sigma \subseteq u(\sigma, \delta)$ with $C \subseteq B$, then $C=B$. Thus, $B$ is a minimal $\sigma$-open set.

(b) Similar to the proof of (a).

(c) Follows from (a) and (b).

Corollary 5. Let $(Y, \sigma, \delta)$ be a BGTS and $B \in \sigma \cap \delta$. Subsequently, the following are equivalent:

(a) B is a minimal s-open set.

(b) B is a minimal u-open set.

Proof. (a) $\Longrightarrow$ (b) Theorem 24 .

(b) $\Longrightarrow$ (a) Theorem 25 (c).

Theorem 26. Let $g:(Y, \sigma) \longrightarrow(Z, \delta)$ be injective and $(\sigma, \delta)$-continuous function. If $B$ is a minimal $\sigma$-open set, such that $g(B) \in \delta$, then $g(B)$ is a minimal $\delta$-open set.

Proof. Since $B \neq \varnothing$, then $g(B) \neq \varnothing$. Suppose $C \subseteq g(B)$ with $C \in \delta-\{\varnothing\}$. Then $g^{-1}(C) \subseteq g^{-1}(g(C))$. Since $g$ is injective, $g^{-1}(g(B))=B$. Because $g$ is $(\sigma, \delta)$-continuous and $C \in \delta$, then $g^{-1}(C) \in \sigma$. As $C \subseteq$ $g(B), g^{-1}(C) \neq \varnothing$. Because $B$ is a minimal $\sigma$-open set, then $g^{-1}(C)=B$. Thus, $B=g\left(g^{-1}(C)\right) \subseteq C$. Hence, $C=g(B)$. 
Corollary 6. Let $g:\left(Y, \sigma_{1}, \delta_{1}\right) \longrightarrow\left(Z, \sigma_{2}, \delta_{2}\right)$ be injective and s-continuous function. If $B$ is a minimal s-open set in $\left(Y, \sigma_{1}, \delta_{1}\right)$ with $g(B) \in \sigma_{2} \cap \delta_{2}$, then $g(B)$ is a minimal s-open set in $\left(Z, \sigma_{2}, \delta_{2}\right)$.

Theorem 27. Let $g:\left(Y, \sigma_{1}, \delta_{1}\right) \longrightarrow\left(Z, \sigma_{2}, \delta_{2}\right)$ be injective and $q$-continuous function. If $B$ is a minimal $q$-open set in $\left(Y, \sigma_{1}, \delta_{1}\right)$ with $g(B) \in q\left(\sigma_{2}, \delta_{2}\right)$, then $g(B)$ is a minimal $q$-open set in $\left(Z, \sigma_{2}, \delta_{2}\right)$.

Proof. Since $B \neq \varnothing$, then $g(B) \neq \varnothing$. Suppose $C \subseteq g(B)$ with $C \in q\left(\sigma_{2}, \delta_{2}\right)-\{\varnothing\}$. Afterwards, $g^{-1}(C) \subseteq g^{-1}(g(B))$. Because $g$ is injective, $g^{-1}(g(B))=B$. As $g$ is $q$-continuous and $C \in q\left(\sigma_{2}, \delta_{2}\right)$, then $g^{-1}(C) \in q\left(\sigma_{2}, \delta_{2}\right)$. Because $C \subseteq g(B), g^{-1}(C) \neq \varnothing$. Since $B$ is a minimal $q$-open set then $g^{-1}(C)=B$. Thus, $B=g\left(g^{-1}(C)\right) \subseteq C$. Hence, $C=f(B)$.

Corollary 7. Let $g:\left(Y, \sigma_{1}, \delta_{1}\right) \longrightarrow\left(Z, \sigma_{2}, \delta_{2}\right)$ be injective and u-continuous function. If $B$ is a minimal $u$-open set in $\left(Y, \sigma_{1}, \delta_{1}\right)$ with $g(B) \in q\left(\sigma_{2}, \delta_{2}\right)$, then $g(B)$ is a minimal u-open set in $\left(Z, \sigma_{2}, \delta_{2}\right)$.

Proof. Corollary 1, Theorem 23 and Theorem 27.

\section{Conclusions}

In this paper, minimal $q$-open set, minimal $u$-open set, and minimal $s$-open sets, $q$-Lindelöf, $u$-Lindelöf, $p$-Lindelöf, $s$-Lindelöf, $q$-countably-compact, $u$-countably-compact, $p$-countably-compact, and s-countably-compact as new concepts of BGTSs are introduced and investigated. In future studies, the following topics could be considered: (1) the definition of fuzzy $u$-open sets and fuzzy $q$-open sets in fuzzy bigeneralized topological spaces; (2) the extension of the two bitopological concepts quasi $\mathcal{N}$-open sets and Quasi $\rightarrow$-open sets, as they appeared in $[19,20]$ to include bigeneralized topological spaces.

Author Contributions: Formal analysis, investigation, and writing-original draft preparation S.A.G. and A.A. All authors have read and agreed to the published version of the manuscript.

Funding: This research received no external funding.

Conflicts of Interest: The authors declare no conflict of interest.

\section{Abbreviations}

The following table summarizes notations used in the paper:

Symbol Interpretation

GT generalized topology

GTS generalized topological space

GTSs generalized topological spaces

BGTS bigeneralized topological space

BGTS bigeneralized topological spaces

q-open quasi-open

$q$-closed quasi-closed

\section{References}

1. Kelly, J.C. Bitopological spaces. Proc. London Math. Soc. 1963, 13, 71-89. [CrossRef]

2. Császár, Á. Generalized topology, generalized continuity. Acta Math. Hungar. 2002, 96, 351-357. [CrossRef]

3. Jayanthi, D. Contra continuity on generalized topological spaces. Acta Math. Hungar. 2012, 137, 263-271. [CrossRef]

4. Tiwari, R.K.; Maitra, J.K.; Vishwakarma, R. Some generalized continuous maps via ideal. Afr. Mat. 2020, 31, 207-217. [CrossRef]

5. Piękosz, A. Locally small spaces with an application. Acta Math. Hungar. 2020, 160, 197-216. [CrossRef]

6. Al Ghour, A. On Slight Omega Continuity and Irresoluteness between generalized topological spaces. Symmetry 2020, 12, 780. [CrossRef] 
7. Chinnaraman, G.; Ramachandran, M.J. One point compactification of generalized topological spaces. Afr. Mat. 2019, 30, 345-353. [CrossRef]

8. Roy, B. On nearly Lindelöf spaces via generalized topology. Proyecciones 2019, 38, 49-57. [CrossRef]

9. Arianpoor, H. On the lattice of principal generalized topologies. Period. Math. Hungar. 2019, 78, 79-87. [CrossRef]

10. Al Ghour, S.; Zareer, W. Some mappings and separation axioms via omega open sets in generalized topological spaces. Nonlinear Stud. 2019, 26, 609-616.

11. Mukharjee, A.; Roy, R.M. On generalized preopen sets. Mat. Stud. 2019, 51, 195-199. [CrossRef]

12. Sini, P. Hereditarily homogeneous generalized topological spaces. Iran. J. Math. Sci. Inform. 2019, 14, 9-18.

13. Tyagi, B.K.; Chauhan, H.V.S. On semi-open sets and feebly open sets in generalized topological spaces. Proyecciones 2019, 38, 875-896. [CrossRef]

14. Piękosz, A.; Wajch, E. Bornological quasi-metrizability in generalized topology. Hacet. J. Math. Stat. 2019, 48, 1653-1666. [CrossRef]

15. Datta, M. Projective bitopological spaces, J. Austral. Math. Soc. 1972, 13, 327-334. [CrossRef]

16. Min, W.K.; Kim, Y.K. Quasi generalized open sets and quasi generalized continuity on bigeneralized topological spaces. Honam Math. J. 2010, 32, 619-624. [CrossRef]

17. Thomas, J.; John, S. $\mu$-compactness in generalized topological spaces. J. Adv. Stud. Topol. 2012, 3, 18-22. [CrossRef]

18. Al Ghour, S.; Al-Omari, A.; Noiri, T. On homogeneity and homogeneity components in generalized topological spaces. Filomat 2013, 27, 1097-1105.

19. Al Ghour, S.; Saleh, H. Quasi $\mathcal{N}$-open sets and related compactness concepts in bitopological spaces. Proyecciones 2018, 37, 491-501. [CrossRef]

20. Al Ghour, S.; Issa, S. On $u$ - $\omega$-open and $q$ - $\omega$-open sets in bitopological spaces. Missouri J. Math. Sci. 2012, 24, 37-53. [CrossRef]

(C) 2020 by the authors. Licensee MDPI, Basel, Switzerland. This article is an open access article distributed under the terms and conditions of the Creative Commons Attribution (CC BY) license (http:/ / creativecommons.org/licenses/by/4.0/). 